\title{
Maintenance of metabolic homeostasis and induction of cytoprotectants and secondary metabolites in alachlor-treated GmGSTU4-overexpressing tobacco plants, as resolved by metabolomics
}

\author{
Christos Kissoudis $^{1,5}$ - Chrysanthi Kalloniati ${ }^{2}$ - Emmanouil Flemetakis ${ }^{2}$. \\ Panagiotis Madesis $^{4} \cdot$ Nikolaos E. Labrou $^{3} \cdot$ Athanasios Tsaftaris $^{1,4}$. \\ Irini Nianiou-Obeidat ${ }^{1}$
}

Received: 2 April 2015/ Accepted: 20 July 2015/Published online: 2 August 2015

(C) Korean Society for Plant Biotechnology and Springer Japan 2015

\begin{abstract}
Herbicides are an invaluable tool for agricultural production scaling up. However, their continuous and intensive use has led to an increased incidence of herbicide resistant weeds and environmental pollution. Plant glutathione transferases (GSTs) are tightly connected with crop and weed herbicide tolerance capacitating their efficient metabolic detoxification, thus GSTs can be biotechnologically exploited towards addressing those issues. However, information on their effects at a "systems" level in response to herbicides is lacking. Here, we aimed to study the effects of the chloroacetanilide herbicide alachlor on the metabolome of wild-type and tobacco plants
\end{abstract}

Electronic supplementary material The online version of this article (doi:10.1007/s11816-015-0364-5) contains supplementary material, which is available to authorized users.

Irini Nianiou-Obeidat

nianiou@agro.auth.gr

1 Department of Genetics and Plant Breeding, School of Agriculture, Forestry and Natural Environment, Aristotle University of Thessaloniki, P.O. Box 261, 54124 Thessaloniki, Greece

2 Laboratory of Molecular Biology, Department of Biotechnology, School of Food, Biotechnology and Development, Agricultural University of Athens, 75 Iera Odos Street, 11855 Athens, Greece

3 Laboratory of Enzyme Technology, Department of Biotechnology, School of Food, Biotechnology and Development, Agricultural University of Athens, 75 Iera Odos Street, 11855 Athens, Greece

4 CERTH, Institute of Applied Biosciences, 6th km CharilaouThermis Road, Thermi, P.O. Box 361, 57001 Thessaloniki, Greece

5 Present Address: Laboratory of Plant Breeding, Wageningen University, Droevendaalsesteeg 1, 6708PB, NL, Wageningen, The Netherlands overexpressing the soybean tau class glutathione transferase GmGSTU4. Alachlor-treated wild-type plants This system, naturally serving the detoxification of endogenous exhibited an abiotic stress-like response with increased abundance of compatible solutes, decrease in TCA cycle intermediates and decreased sugar and amino acid content. Transgenic plants responded distinctly, exhibiting an increased induction of abiotic stress responsive metabolites, accumulation of secondary metabolites and its precursors, and metabolic detoxification by-products compared to wild-type plants. These results suggest that the increased metabolic capacity of $\mathrm{GmGSTU} 4$ overexpressing plants is accompanied by pleiotropic metabolic alterations, which could be the target for further manipulation in order to develop herbicide resistant crops, plants with increased phytoremediation potential, as well as efficient management of non-target site, GST induced, herbicide resistance in weeds.

Keywords Herbicide resistance $\cdot$ Non-target site resistance $\cdot$ Secondary metabolism $\cdot$ Glutathione $\cdot$ Abiotic stress $\cdot$ Phytoremediation

\section{Introduction}

Herbicide applicability in crops is vastly dependent on their selectivity, being able to control weeds while not affecting the crop itself. Herbicide selectivity is either based on target insensitivity (target site resistance) (Devine and Shukla 2000) or increased metabolic capacity which leads to herbicide catabolism and inactivation through a threephase detoxification system (non-target site resistance) (Yuan et al. 2007). This system, naturally serving the detoxification of endogenous toxins and degradation of 
oxidative stress by-products (Dixon et al. 2010), comprises three major enzymatic systems functioning subsequently: cytochrome P450 monooxygenases (CYP450s), GSTs and/ or glycosyltransferases (GTs), and ATP-binding cassette (ABC) transporters (Powles and Yu 2010), whose augmented activity and/or specificity results in accelerated herbicide metabolism (Délye 2013). GSTs, the major phase II detoxification enzymes, catalyse the conjugation of the xenobiotic with glutathione, increasing its hydrophilicity thus facilitating its excretion from the cytoplasm and its compartmentation in the vacuole by $\mathrm{ABC}$ transporters in phase III (Cummins et al. 2011).

Chloroacetanilides comprise a class of herbicides that inhibit very long chain fatty acid (VLCFA) synthetase (Böger 2003), the condensing starter enzyme of the elongase system for the biosynthesis of VLCFA, fatty acids with chain lengths of 20 or more carbons. VLCFAs in plants are important biological components of lipids present in various cell membranes and the cuticular waxes (Bach and Faure 2010). Chloroacetanilide herbicides are used in large scale for the control of annual grasses and broadleaf weeds. This has resulted in various unintended effects such as the development of weed resistance (Cummins et al. 2013). It has also resulted in environmental pollution with many studies reporting increased residual concentration of chloroacetanilides in off-target sites and ground water, potentially resulting in toxic side effects to non-target organisms (Abrantes et al. 2008; He et al. 2012).

GSTs biotechnological exploitation has major implications in facing these issues: GSTs can be exploited to enhance herbicide selectivity in crops, either through their transgenic overexpression (Milligan et al. 2001), or primed up regulation by the exogenous chemical application (safeners) (Behringer et al. 2011). Moreover, their targeted chemical inactivation can be deployed to seize weed resistance resulting from increased GST activity (Cummins et al. 2013). Specific GST overexpression in plants can enhance their metabolic capacity for the detoxification of herbicide and other organic compounds or even environmental pollution through phytoremediation (Kawahigashi 2009; Zhang and Liu 2011).

The availability of multiple-omics technologies has enabled the comprehensive dissection of phenotypes in the molecular level, enabling better understanding of the underlying biological processes which could lead to their manipulation (Mochida and Shinozaki 2011). Among other-omics, metabolomics approaches are increasingly being engaged for research purposes towards the identification and classification of agrochemical's mechanism of action (Aliferis and Jabaji 2011; Trenkamp et al. 2009) as well as for the dissection of mechanisms leading to herbicide resistance (Délye 2013). As metabolites are the ultimate products of gene expression and enzymatic reactions, their simultaneous monitoring can reveal perturbations in biochemical and metabolic networks critical for the phenotypic expression, that can lead to further manipulation through metabolic engineering (Farré et al. 2014).

In previous studies, we comprehensively characterized GmGSTU4, a tau class GST form soybean, for its structural and catalytic properties. GmGSTU4 exhibits increased catalytic activity towards the herbicides alachlor and fluorodifen, as well as increased glutathione peroxidise (GPOX) activity towards fatty acid hydroxyperoxides (Axarli et al. 2009). GmGSTU4 overexpression in tobacco plants resulted in greater tolerance towards both alachlor and fluorodifen (Benekos et al. 2010) and increased salt stress tolerance which was accompanied by significant alterations in the transgenic plant metabolome towards maintaining metabolic homeostasis and augmenting the production of stress ameliorating metabolites (Kissoudis et al. 2015).

Non-target site herbicide resistance is characterized not only by an increased rate of herbicide catabolism but also pleiotropic alterations (Cummins et al. 2013; Vila-Aiub et al. 2005). Moreover, increased evidence points to multiple roles of GSTs in endogenous metabolism (Dixon et al. 2010). Therefore, aiming to investigate the responses leading to increased resistance to alachlor in GmGSTU4 overexpressing plants in comparison to wild-type (WT) plants, we employed gas chromatography/mass spectrometry (GC-MS) metabolomics. The results revealed pleiotropic alterations in plant metabolism in WT plants as a consequence of alachlor toxicity, and a distinct response in transgenic plants, with apparent metabolic signatures of the increased detoxification capacity provided by GmGSTU 4 as well as greater accumulation of secondary metabolites and their precursors. These findings can be further exploited to efficiently manage non-target site resistance in weeds, as well as the generation of crops exhibiting increased robustness to herbicides and plant with greater phytoremediation capacity towards chloroacetanilides.

\section{Materials and methods}

\section{Plant material and alachlor treatment}

Wild-type (WT) tobacco cv. Basmas and transgenic line L3 overexpressing the soybean tau class glutathione transferase GmGSTU4 were grown in vitro as described (Benekos et al. 2010). Line L3 as shown previously (Benekos et al. 2010) exhibited the greater increase in GST and GPOX activity and tolerance to herbicide treatments and as it carried a single transgene insert, it was selected and used in the experiments presented here (hereafter mentioned as GST-OX). 
Two-week-old WT and transgenic plants were transferred to Magenta GA-7 vessels containing MurashigeSkoog (MS) medium supplemented with the herbicide alachlor as a commercial formulation (ALANEX ${ }^{\circledR} 48 \mathrm{EC}$ ), at an active ingredient concentration of $3 \mathrm{mg} / \mathrm{L}$, while control plants of both genotypes were similarly grown in MS medium without alachlor. After 20 days of growth, the 2nd and 3rd leaf from top were sampled and after lyophilization, were stored at $-80{ }^{\circ} \mathrm{C}$.

\section{Metabolite extraction, derivatisation and GC-MS analysis}

Approximately, $10 \mathrm{mg}$ dry weight of tissue was ground in liquid nitrogen. Ground samples were extracted with $380 \mu \mathrm{L}$ methanol and $20 \mu \mathrm{L}$ ribitol in methanol $\left(0.2 \mathrm{mg} \mathrm{mL}^{-1}\right)$. Samples were incubated at $70{ }^{\circ} \mathrm{C}$ for 15 min with continuous shaking. The addition of $200 \mu \mathrm{L}$ chloroform was followed by a further incubation at $37{ }^{\circ} \mathrm{C}$ for $5 \mathrm{~min}$ under continuous shaking. After addition of

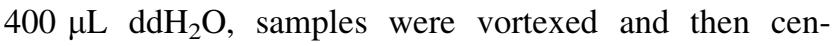
trifuged at 18,000 $\mathrm{g}$ for $5 \mathrm{~min}$ at room temperature. The aqueous phase containing the polar metabolite fraction was transferred into new eppendorf tubes and dried by nitrogen gas. For derivatisation, dried samples were re-suspended in $25 \mu \mathrm{l}$ methoxyamine- $\mathrm{HCl}$ (MOX, $20 \mathrm{mg} \mathrm{mL}^{-1}$ in pyridine), and incubated at $30{ }^{\circ} \mathrm{C}$ for 90 min with continuous gentle agitation. This was followed by addition of $50 \mu \mathrm{l}$ of $\mathrm{N}$-methyl- $\mathrm{N}$-(trimethylsilyl)-trifluoroacetamide (MSTFA) and incubated at $37^{\circ} \mathrm{C}$ for $30 \mathrm{~min}$ with continuous gentle agitation. Finally, $10 \mu \mathrm{l}$ of n-alkane mix were added for determination of retention indexes. GC-MS measurements were performed in a HP6890 GC coupled to a HP 5973 MS.

\section{Data analysis}

For all treatments tested, five biological replications were performed. The chromatograms were evaluated automatically using the AMDIS software and metabolites were identified using the Golm metabolome database (Kopka et al. 2005; Schauer et al. 2005). Results were expressed as a response that corresponds to the ratio between the areas of the target metabolite divided by the area of the reference metabolite (ribitol, $m / z$ 319) and reported relative to the dry weight.

Principal component analysis (PCA) was performed on the relative metabolite concentration data using the Unscrambler 9.5 software (CAMO Software Inc., NJ, USA). Statistically significant differences between genotypes and treatments for each metabolite were validated by the Student's $t$ test $(P \leq 0.05)$.

\section{Results}

In our previous study, alachlor tolerance of GmGSTU4 overexpressing tobacco plants was evaluated at herbicide concentrations that completely inhibited WT plant growth (Benekos et al., 2010). To identify the effects of alachlor on WT plants metabolism, and to compare them with tobacco plants overexpressing GmGSTU4, plants were grown in $3 \mathrm{mg} / \mathrm{L}$ alachlor concentration (which corresponds to the higher dose applied in field conditions, Kawahigashi et al., 2005) as compared to 7.5 and $15 \mathrm{mg} / \mathrm{L}$ which are 7- and 14-fold higher than the optimal field dose (Benekos et al. 2010). At this concentration transgenic plants showed high level of tolerance while WT plants could maintain part of their growth (Supp. Figure 1). This allowed the appropriate comparison of alachlor responses between the two genotypes (Aliferis and Chrysayi-Tokousbalides 2011), devoid of potential unintended effects due to acute toxicity of alachlor at higher concentrations on WT plants as observed in our previous experiments (Benekos et al. 2010). Thus, mechanisms potentially linked to the increased tolerance of the transgenic plants to alachlor could be more appropriately identified.

Analysis of leaf extracts, carried out by GC/MS, identified a total of 98 polar metabolites many of which were filtered out due to low abundance and inconsistent presence to a remaining 86 metabolites (Supp. Table 1). On this basis, we examined metabolic alterations in WT and GSTOX plants after alachlor treatment and compared them for the identification of metabolic signatures of both alachlor toxicity and increased alachlor tolerance conferred by GmGSTU4.

\section{Metabolic responses of wild-type tobacco plants under alachlor treatment}

WT tobacco plants metabolism was significantly affected by alachlor, with the abundance of about $30 \%$ (27/87) of the metabolites identified significantly altered in comparison to control conditions. These metabolites spanned all the different functional categories covered by the GC-MS analysis, indicating pleiotropic effects of alachlor on plant metabolism. Metabolite data were loaded for PCA to identify the pattern of metabolic changes and the contribution of the underlying metabolites. The two highest ranking principal components accounted for $72 \%$ of the total variance. PCA revealed a distinct fingerprint of the WT plants metabolome grown in $3 \mathrm{mg} / \mathrm{L}$ alachlor in comparison to non-stress conditions. Differences between treatments were resolved by PC1 with non-stressed plants being on the positive side while fingerprints of alachlortreated plants drifted towards the negative side of PC1. The 
metabolic loadings in principal component 1 ( $\mathrm{PC} 1)$ were examined and revealed high positive values for the hexoses glucose, fructose and mannose and negative values for phosphoric acid, proline and sucrose. This indicated that differences can be attributed to the reduction in the concentration of those hexoses and the accumulation of phosphate, proline and sucrose in alachlor-treated WT plants (Fig. 1; Supp. Table 2).

Furthermore, individual metabolite alterations between treatments were compared using the $t$ test $(p \leq 0.05)$. While the directionality of all the above-mentioned alterations was the same, significant differences were only observed for proline and mannose. A metabolic trend was observed as the concentration of most of the amino acids and organic acids was decreased, though few differences were identified as significant. The concentration of 27 metabolites was significantly altered, of which 8 increased and 19 decreased (Table 1). Metabolites whose abundance
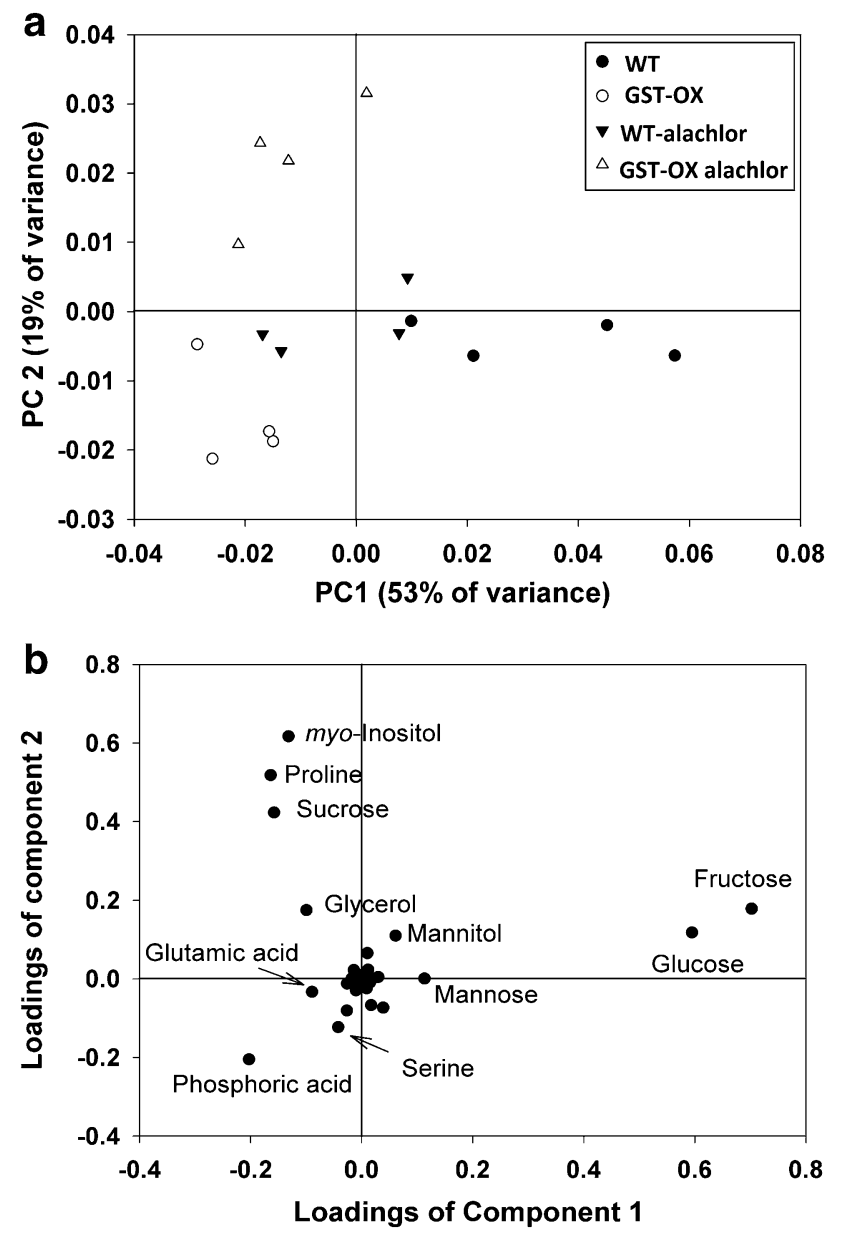

Fig. 1 Principal component analysis a of leaf metabolites identified in WT and $G m$ GSTU4 overexpressing tobacco plants grown in vitro for 20 days in plain MS medium or supplemented with $3 \mathrm{mgL}$ alachlor. Principal components (PC) 1 and 2 cumulatively account for $72 \%$ of the variance in the data. b Metabolite loadings plot for PC1 and PC2. Loading values for specific metabolites are indicated significantly increased after alachlor treatment were the amino acid proline and its derivative hydroxy-proline (3.1and 4.8-fold increase respectively), the photorespiration intermediate glycolic acid (4.7-fold), as well as the sugar alcohol mannitol (76.5-fold), hydroquinone (18.3-fold) and myo-inositol (2.1-fold), which commonly serve as osmolytes/osmoprotectants under abiotic stress. On the other hand, a significant decrease was observed in the concentration of the aromatic amino acids tryptophan and tyrosine (18- and 5-fold respectively) precursors of several families of secondary metabolites, as well as isoleucine (4.9-fold) which is linked to pyruvic acid. The tricarboxylic acid (TCA) cycle intermediates pyruvic acid and succinic acid decreased 3.7- and 2.4-fold, respectively. Among others, the non-structural disaccharides lactose and maltose, the latter involved in starch remobilization, decreased 10.2and 6.7-fold respectively, while mannose and its amino sugar mannosamine, precursors of cell wall constituents, were not detectable after alachlor application.

\section{Metabolic responses of GST-OX tobacco plants under alachlor treatment}

The abundance of a significant number of metabolites of GST-OX plants was affected as well by alachlor (26 out of 87), but the response was distinct in comparison to WT under alachlor stress, reflecting the increased tolerance of GST-OX plants through the capacitation of alachlor catabolism. This is reflected in the PCA analysis, where a distinct fingerprint was observed for alachlor-treated GSTOX plants in comparison to WT (Fig. 1). The metabolic alterations after alachlor treatment in GST-OX plants were resolved by $\mathrm{PC} 2$, with non-treated plants residing on the negative side of $\mathrm{PC} 2$, while alachlor treatment resulted in a shift to the positive side of PC2. Metabolites with highest positive values were myo-inositol, proline and glycerol, reported to be functioning as osmoprotectants during abiotic stress, as well as the hexoses glucose and fructose and the disaccharide sucrose, indicating increased accumulation under alachlor treatment (Fig. 1; Supp. Table 2).

In line with the distinct response of GST-OX plants to alachlor, 19 metabolites significantly increased (14 unique to GST-OX plants), while 7 decreased (3 unique to GSTOX plants) (Fig. 2). Among the commonly induced metabolites in both WT and GST-OX are metabolites that function as compatible solutes and involved in abiotic stress responses (hydroquinone, myo-inositol, proline, 4-hydroxy-L-proline). Compounds that uniquely accumulated in GST-OX plants were cellobiose and malonic acid (8.6- and 4.2-fold), precursors of cell wall and fatty acid biosynthesis, respectively, the nitrogen assimilation product allantoin (4.9-fold), nicotinamide, a (nicotinamide adenine dinucleotide) NAD biosynthesis intermediate 
Table 1 Metabolites that were significantly altered $(p<0.05)$ in WT and GST-OX plants grown in $3 \mathrm{mg} / \mathrm{L}$ alachlor in comparison to control conditions

\begin{tabular}{|c|c|c|c|}
\hline \multicolumn{2}{|l|}{ WT alachlor/WT control } & \multicolumn{2}{|c|}{ GST-OX alachlor/GST-OX control } \\
\hline Metabolites & Fold change & Metabolites & Fold change \\
\hline Mannitol & 76.5 & Glycerol & 21.0 \\
\hline Hydroquinone & 18.3 & Hydroquinone & 8.9 \\
\hline Trans-4-hydroxy-L-proline & 4.8 & Proline & 8.6 \\
\hline Glycolic acid & 4.7 & Cellobiose & 8.6 \\
\hline Proline & 3.1 & Allantoin & 4.9 \\
\hline Myo-inositol & 2.1 & Malonic acid & 4.2 \\
\hline Palatinose & stress sp. & Nicotinamide & 4.0 \\
\hline \multirow[t]{3}{*}{ Resorcinol } & stress sp. & Nornicotine & 3.8 \\
\hline & & Myo-inositol & 3.8 \\
\hline & & Pipecolic acid & 3.6 \\
\hline Putrescine & -1.7 & Mimosine & 3.2 \\
\hline b-cyano-L-alanine & -1.8 & Caffeic acid & 2.7 \\
\hline Galactinol & -2.2 & Acetol & 2.3 \\
\hline Succinic acid & -2.4 & Sucrose & 1.4 \\
\hline Urea & -2.8 & Resorcinol & stress sp. \\
\hline Porphine & -3.0 & Sophorose & stress sp. \\
\hline Pyruvic acid & -3.7 & Trans-4-hydroxy-L-proline & stress sp. \\
\hline Isoleucine & -4.9 & Tyramine & stress sp. \\
\hline Tyrosine & -5.0 & Loganin & stress sp. \\
\hline Maltose & -6.7 & & \\
\hline Lactose & -10.2 & & \\
\hline Tryptophan & -18.0 & Serine & -1.7 \\
\hline Melibiose & -25.4 & b-cyano-L-alanine & -1.7 \\
\hline Nicotinamide & ctr. sp. & Succinic acid & -1.9 \\
\hline Maleamic acid & ctr. sp. & Threonine & -2.0 \\
\hline Mannose & ctr. sp. & Urea & -2.2 \\
\hline N-acetyl-D-mannosamine & ctr. sp. & Putrescine & -2.2 \\
\hline Tartaric acid & ctr. sp. & D-glucose-6-phosphate & -3.5 \\
\hline Tyramine & ctr. sp. & & \\
\hline
\end{tabular}

(fourfold) and acetol (2.3-fold) a product of methylglyoxal and reactive carbonyl detoxification. Moreover, several secondary metabolites accumulated, such as nornicotine, mimosine, caffeic acid, loganin and tyramine. Metabolites that were uniquely decreased in GST-OX plants were the amino acids serine and threonine (1.7- and 2-fold, respectively) and D-glucose-6-phosphate (3.5-fold).

\section{Comparison of WT and GST-OX tobacco plants metabolome under alachlor treatment}

The contrasting responses of WT and GST-OX tobacco plants to alachlor resulted in distinct metabolic fingerprints as is evident from the PCA analysis. To identify metabolic markers that are linked to $G m G S T U 4$-induced resistance to alachlor we directly compared the metabolic profiles of WT and GST-OX plants under alachlor treatment. In this way, metabolic alterations resulting from the constitutive
GmGSTU4 expression (Supp. Table 1) are also taken into account. The results reflect partly the contrasting response observed in WT and GST-OX plants, with the latter exhibiting a potentiated secondary metabolism (higher abundance of tryptophan and tyrosine, and secondary metabolites), higher concentration of proline and proline catabolic compounds, as well as other compounds with protective functions (glycerol and hydroquinone), higher lactose and acetol accumulation but lower manittol and glycolic acid concentration. Moreover, many metabolic alterations resulting from the constitutive GmGSTU4 expression in non-stress conditions (Supplementary Table 1), discussed in our previous study (Kissoudis et al. 2015), persisted after alachlor treatment, as is evident by the higher abundance of acetyl-L-serine, a precursor of glutathione biosynthesis, and glycerol 1-phosphate as well as lower concentration of the hexoses glucose and fructose (Table 2). 


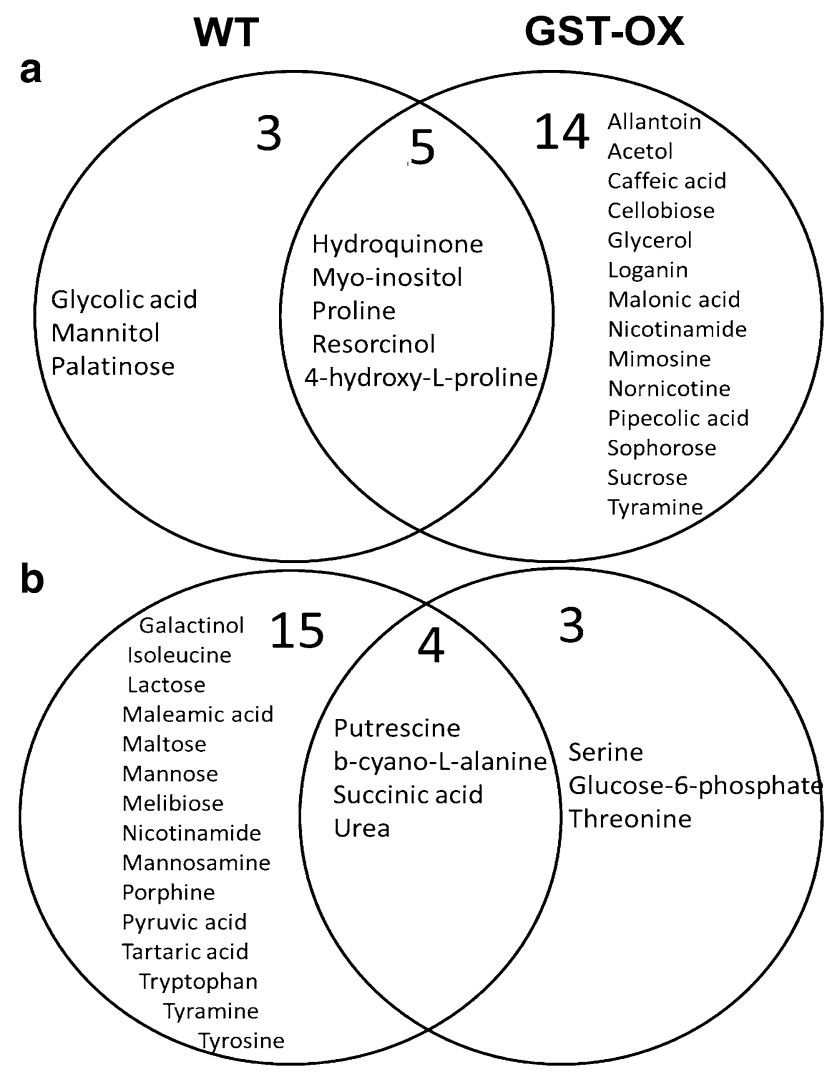

Fig. 2 Venn diagram representation of metabolites commonly or differentially increased (a) or decreased (b) in the leaves of WT and transgenic tobacco plants grown in vitro for 20 days in MS medium supplemented with $3 \mathrm{mg} / \mathrm{L}$ alachlor in comparison to plain MS medium

\section{Discussion}

The identification of GSTs as a crucial component of nontarget side resistance against herbicides enabled their deployment through biotechnological approaches for building of herbicide resistance in crops (Cummins et al. 2011), as well as for enhanced plant phytoremediation capacity (Karavangeli et al. 2005). Yet, very little is known on the metabolic alterations related to herbicide resistance in the genetically modified plants overexpressing GSTs. Metabolite monitoring can enable the identification of biomarkers for herbicide toxicity as well as metabolic signatures of resistance (Aliferis and Chrysayi-Tokousbalides 2011).

Metabolomics analysis revealed pleiotropic effects of alachlor on WT tobacco plants metabolism. The metabolic alterations after alachlor treatment resembled a response to an abiotic stressor, as the accumulated metabolites like mannitol, proline and hydroquinone are commonly identified as induced metabolites after abiotic stress, serving as osmoprotectants and (reactive oxygen species) ROS scavengers (Krasensky and Jonak 2012). Indications of
Table 2 Differentially accumulated metabolites $(p<0.05)$ in WT and and GST-OX plants grown in $3 \mathrm{mg} / \mathrm{L}$ alachlor

\begin{tabular}{|c|c|}
\hline \multicolumn{2}{|c|}{ GST-OX alachlor/WT alachlor } \\
\hline Metabolites & Fold difference \\
\hline Lactose & 23.8 \\
\hline Melibiose & 15.3 \\
\hline Glycerol & 9.4 \\
\hline Tryptophan & 8.2 \\
\hline Allantoin & 5.7 \\
\hline Pyrrole-2-carboxylic acid & 5.3 \\
\hline Acetol & 4.2 \\
\hline Malonic acid & 3.7 \\
\hline Isoleucine & 3.6 \\
\hline Acetyl-L-serine & 3.6 \\
\hline Tyrosine & 3.2 \\
\hline Porphine & 3.1 \\
\hline Mimosine & 2.8 \\
\hline Resorcinol & 2.6 \\
\hline Hydroquinone & 2.6 \\
\hline Cellobiose & 2.5 \\
\hline Maltose & 2.3 \\
\hline Glycerol 1-phosphate & 2.2 \\
\hline Proline & 1.7 \\
\hline Trans-4-hydroxy-L-proline & 1.7 \\
\hline Nicotinamide & GST-ox sp. \\
\hline Loganin & GST-ox sp. \\
\hline Maleamic acid & GST-ox sp. \\
\hline Mannose & GST-ox sp. \\
\hline$N$-acetyl-D-mannosamine & GST-ox sp. \\
\hline Tartaric acid & GST-ox sp. \\
\hline Tyramine & GST-ox sp. \\
\hline Glucose & -2.0 \\
\hline Fructose & -2.5 \\
\hline Glycolic acid & -4.4 \\
\hline$\beta$-thiogalactopyranoside & -4.8 \\
\hline Mannitol & -25.0 \\
\hline
\end{tabular}

photorespiration up regulation reinforce this conclusion, as it results in ROS production (Voss et al. 2013). Similarly, transcriptome analysis of Arabidopsis plants treated with another chloroacetanilide herbicide, flufenacet, indicated a marked up regulation of wound responsive genes (LecheltKunze et al. 2003), while treatment of Triticum tauschii seedlings with dimethenamid resulted in the induction of several abiotic stress responsive proteins (Zhang et al. 2007). Furthermore, the arabidopsis mutant pasticcino, defective in VLCFA, exhibits aberrant transcriptional alterations with several genes induced being involved in salt and dehydration stress response (Nobusawa et al. 2013). 
Alachlor treatment on WT tobacco plants resulted in an apparent deregulation of central metabolism, as levels of sugars, amino acids and TCA cycle intermediates were in general decreased, indicating a perturbation of the core metabolism that further lad the WT plants to succumb to the herbicide. Furthermore, the decreased concentration of metabolites serving as precursors of cell wall biosynthesis indicated a concerted down regulation of growth. Alterations in carbohydrate metabolism have also been reported for the acetolactate synthase (ALS) inhibiting herbicides and the 5-enolpyruvylshikimate-3-phosphate synthase (EPSPS) inhibiting herbicide glyphosate that target branched chain and aromatic acid biosynthesis, respectively (Orcaray et al. 2012; Zabalza et al. 2011). Therefore, central metabolism perturbations are integral to the toxicity mechanisms of herbicides with unrelated mechanism of action and should be further considered in toxicological and environmental studies (Serra et al. 2013).

In addition, alachlor resulted in an apparent down regulation of secondary metabolism in WT plants with severe reduction in the abundance of tryptophan and tyrosine, major substrates for the production of secondary metabolites (Tzin and Galili 2010). Similar results were obtained in early studies of alachlor activity in maize and sorghum, where its application resulted in decreased lignin content and anthocyanin accumulation (Lydon and Duke 1989). The effects of alachlor on secondary metabolism should be further evaluated, especially in relation to ecotoxicological studies, as secondary metabolites are important for plant responses to biotic and abiotic stresses (Bednarek 2012), as their perturbation may limit the adaptive potential of the non-target organisms. It should be noted here though that our approach utilizing GC-MS, cannot comprehensively cover non-polar secondary metabolites, and should be further coupled with Liquid chromatography/Mass spectrometry (LC-MS) to provide a broader picture of the secondary metabolism regulation including specific pathways and compounds that are differentially regulated (Matsuda et al. 2009).

Capacitation of alachlor metabolism by GmGSTU4 overexpression, resulting in increased tolerance of transgenic tobacco plants to the herbicide, was accompanied by a distinct metabolic response, reversing or cancelling the alterations observed in WT plants and inducing in greater magnitude protective and detoxification pathways. Similarly to WT, metabolites related to abiotic stress response were induced; however, either the magnitude of accumulation was higher (e.g. proline) or they were uniquely accumulated (glycerol). Most of these metabolites are shown to contribute to abiotic stress tolerance (Eastmond 2004; Sharma et al. 2011). Therefore, induced abiotic stress tolerance might be a part of the increased alachlor tolerance of transgenic plants, protecting against its non-target cytotoxic effects. Up regulation of abiotic/oxidative stress tolerance genes has been observed in resistant weed ecotypes to herbicides with different modes of action (Duhoux et al. 2015; Gaines et al. 2014). These observations have broader implications on the mechanisms involved in nontarget site resistance against multiple herbicides (Cummins et al. 2013; Délye 2013). Interestingly, the same metabolites were differentially up regulated in GmGSTU4 overexpressing after salt stress treatment (Kissoudis et al. 2015) hinting a potential regulatory/signalling role of GmGSTU4 for their accumulation under stress. Further indications for enhanced cytoprotection are supported by the accumulation of acetol, a product of toxic aldehyde detoxification, to which GSTs are directly enzymatically involved (Fujita and Hossain 2003), with GmGSTU4 in particular exhibiting significant enzymatic activity (Axarli et al. 2009).

The accumulation of secondary metabolites in transgenic plants after alachlor treatment was in stark contrast with the response of WT plants. This pattern has significant similarity with the result obtained after constitutive overexpression in Arabidopsis of a black-grass GST conferring broad spectrum herbicide resistance, which lead to increased accumulation of secondary metabolites (He et al. 2012). Previous research has shown a role for GSTs in flavonoid metabolism/transport, as specific GSTs can bind to flavonoids and other secondary metabolites, potentially protecting unstable intermediates from auto-oxidation (Chan and Lam 2014; Dixon et al. 2010). GmGSTU4 was shown to possess an L-site (Axarli et al. 2009), which can function in a similar way and therefore stabilizing secondary metabolite intermediates, reducing their turnover and resulting in their greater accumulation. A role of secondary metabolites in the protection against herbicide toxicity comes from observations after application of safeners, which results in the up regulation of genes encoding enzymes of the secondary metabolism as well the accumulation of secondary metabolites (Behringer et al. 2011; Cummins et al. 2006; Skipsey et al. 2011). Enhanced accumulation of secondary metabolites through transgenic manipulation was shown to confer general stress robustness (Nakabayashi et al. 2014; Zhang et al. 2013), providing further evidence for their beneficial properties under stress.

The differential metabolic responses of WT and GmGSTU4 overexpressing plants share a significant similarity with observations made at the protein level in Triticum tauschii seedlings treated with a chloroacetanilide herbicide alone and in combination with a safener which induced resistance (Zhang et al. 2007). While herbicide treatment alone induced only stress responsive proteins, the safener co-treatment resulted in the concerted up regulation of detoxification pathways, including many GST proteins, as well as jasmonic acid/oxylipin and secondary metabolism pathways (Zhang et al. 2007). The convergence of 
these observations suggests that GSTs are merely a passive component of the herbicide detoxification mechanism, and their timely induction after herbicide treatment may not only be directly linked to enhanced detoxification capacity but also the sensitization of multiple cytoprotective mechanisms. This is further supported by our findings that constitutive GmGSTU4 overexpression leads to alteration in tobacco plants metabolism even under non-stress conditions (Kissoudis et al. 2015). Many of these alterations were maintained even after alachlor treatment and may have contributed to increased tolerance to alachlor. Therefore, it appears that GmGSTU4 overexpression results in a stress-priming effect that, except for the direct metabolic capacity of GmGSTU4 towards alachlor, is sensitizing multiple detoxification/defence components that help cope with toxicity. How GmGSTU4 leads to these alterations remains elusive, although there is evidence for altered redox homeostasis as evident by the altered accumulation of glutathione and ascorbic acid biosynthesis precursors (Kissoudis et al. 2015). Altered glutathione homeostasis in gstu17 mutants in Arabidopsis resulted in greater abscisic acid (ABA) accumulation and abiotic stress tolerance (Chen et al. 2012). Moreover, GSTs appear to be involved in oxylipin signalling, important regulators of redox homeostasis under stress conditions (Park et al. 2013), through their direct binding (Dixon and Edwards 2009), and as GmGSTU4 has ligandin functions through its L-site, this possibility cannot be excluded.

In conclusion, metabolomics analysis indicated that alachlor toxicity in WT tobacco plants is translated as an abiotic stress response accompanied by the down regulation of the primary metabolism and reduced accumulation of secondary metabolites precursors. GmGSTU4 overexpression in part reversed these responses, as well as leads to greater induction of protective and detoxification responses, including up regulation of the secondary metabolism (Fig. 3). These findings provide further insights on alachlor toxicity on weeds as well as non-target organisms and increase our understanding for GST induced chloroacetanilide herbicide resistance. Moreover, these results can enable both a more efficient management of herbicide resistant weeds, through chemical or genetic (RNAi) inhibition of the underlying enzymes controlling these metabolic processes ( $\mathrm{Yu}$ and Powles 2014), the efficient deployment of chloroacetanilide resistant crops as well as plants targeted for herbicide phytoremediation in contaminated sites. Current approaches employ the engineering of single or multiple components of the three-phase detoxification system of xenobiotics (Kawahigashi et al. 2005; Zhang and Liu 2011). On top of these approaches, manipulation for antioxidant/osmoprotectant metabolite accumulation as well as enhanced detoxification of toxic byproducts of oxidative stress such as alkenals and aldehydes

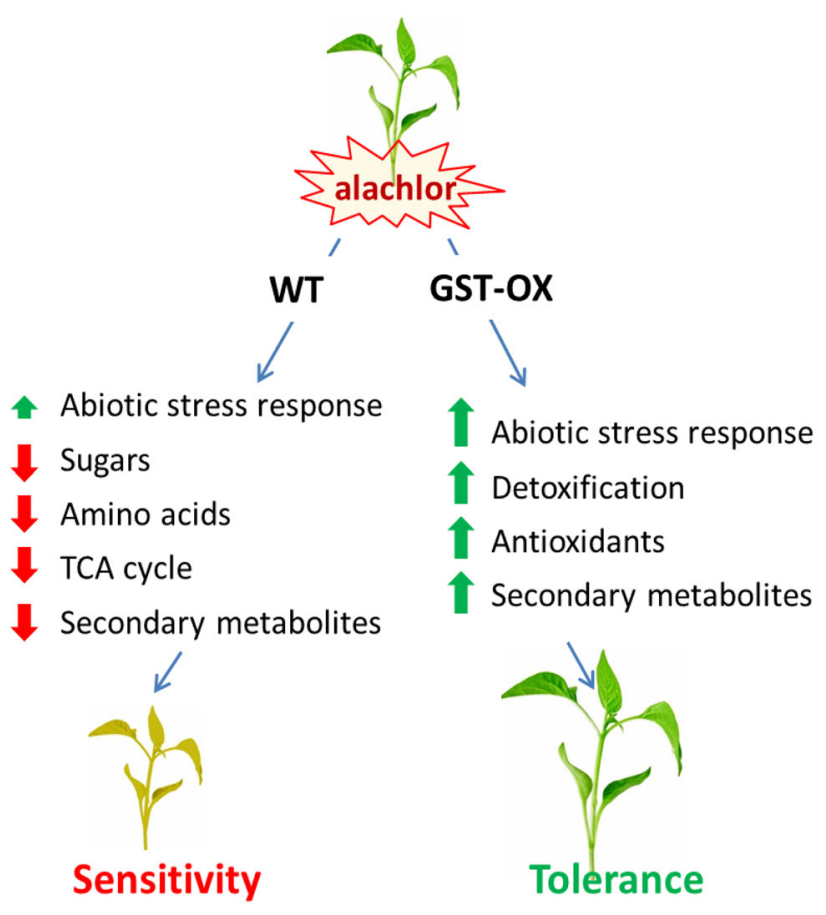

Fig. 3 Schematic representation of the differential metabolic regulation of WT and GST-OX tobacco plants underlying the increased tolerance to alachlor after GmGSTU4 overexpression in GST-OX plants

through increasingly sophisticated approaches (Aharoni and Galili 2011; Shachar-Hill 2013) has the potential to increase the robustness of crops against the indirect effects of herbicide application. The results of the metabolomics analysis could potentially serve as biomarkers for the discovery of plant species or even individuals resistant to stresses with important implications in plant breeding projects—of course this remains to be further evaluated. Further exploration of these pathways and identification of causality relationships with herbicide resistance has the potential to contribute in increasing the sustainability of agricultural practices and the robustness of crops.

Acknowledgments Christos Kissoudis is supported by "Alexander S. Onassis" Public Benefit Foundation scholarship. The authors (NEL, PM, INO) would acknowledge the Ministry of Education, Lifelong Learning and Religious Affairs for the financial assistance provided under the action THALES: "Glutathione transferases: multifunctional molecular tools in red and green biotechnology". This action falls under the Operational Programme "Education and Lifelong Learning"' and is co-funded by the European Social Fund and National Resources.

\section{References}

Abrantes N, Pereira R, Soares AMVM, Gonçalves F (2008) Evaluation of the ecotoxicological impact of the pesticide Lasso ${ }^{\circledR}$ on non-target freshwater species, through leaching from nearby 
agricultural fields, using terrestrial model ecosystems. Water Air Soil Pollut 192:211-220

Aharoni A, Galili G (2011) Metabolic engineering of the plant primary-secondary metabolism interface. Curr Opin Biotechnol 22:239-244

Aliferis KA, Chrysayi-Tokousbalides M (2011) Metabolomics in pesticide research and development: review and future perspectives. Metabolomics 7:35-53

Aliferis KA, Jabaji S (2011) Metabolomics-a robust bioanalytical approach for the discovery of the modes-of-action of pesticides: a review. Pestic Biochem Physiol 100:105-117

Axarli I, Dhavala P, Papageorgiou AC, Labrou NE (2009) Crystallographic and functional characterization of the fluorodifeninducible glutathione transferase from Glycine max reveals an active site topography suited for diphenylether herbicides and a novel L-site. J Mol Biol 385:984-1002

Bach L, Faure JD (2010) Role of very-long-chain fatty acids in plant development, when chain length does matter. Comptes Rendus Biol 333:361-370

Bednarek P (2012) Chemical warfare or modulators of defence responses-the function of secondary metabolites in plant immunity. Curr Opin Plant Biol 15:407-414

Behringer C, Bartsch K, Schaller A (2011) Safeners recruit multiple signalling pathways for the orchestrated induction of the cellular xenobiotic detoxification machinery in Arabidopsis. Plant Cell Environ 34:1970-1985

Benekos K, Kissoudis C, Nianiou-Obeidat I, Labrou N, Madesis P, Kalamaki M, Makris A, Tsaftaris A (2010) Overexpression of a specific soybean GmGSTU4 isoenzyme improves diphenyl ether and chloroacetanilide herbicide tolerance of transgenic tobacco plants. J Biotechnol 150:195-201

Böger P (2003) Mode of action for chloroacetamides and functionally related compounds. J Pestic Sci 28:324-329

Chan C, Lam H-M (2014) A putative lambda class glutathione S-transferase enhances plant survival under salinity stress. Plant Cell Physiol 55:570-579

Chen JH, Jiang HW, Hsieh EJ, Chen HY, Chien CT, Hsieh HL, Lin TP (2012) Drought and salt stress tolerance of an arabidopsis glutathione S-transferase U17 knockout mutant are attributed to the combined effect of glutathione and abscisic acid. Plant Physiol 158:340-351

Cummins I, Brazier-Hicks M, Stobiecki M, Frański R, Edwards R (2006) Selective disruption of wheat secondary metabolism by herbicide safeners. Phytochemistry 67:1722-1730

Cummins I, Dixon DP, Freitag-Pohl S, Skipsey M, Edwards R (2011) Multiple roles for plant glutathione transferases in xenobiotic detoxification. Drug Metab Rev 43:266-280

Cummins I, Wortley DJ, Sabbadin F, He Z, Coxon CR, Straker HE, Sellars JD, Knight K, Edwards L, Hughes D, Kaundun SS, Hutchings SJ, Steel PG, Edwards R (2013) Key role for a glutathione transferase in multiple-herbicide resistance in grass weeds. Proc Natl Acad Sci USA 110:5812-5817

Délye C (2013) Unravelling the genetic bases of non-target-site-based resistance (NTSR) to herbicides: a major challenge for weed science in the forthcoming decade. Pest Manag Sci 69:176-187

Devine MD, Shukla A (2000) Altered target sites as a mechanism of herbicide resistance. Crop Prot 19:881-889

Dixon DP, Edwards R (2009) Selective binding of glutathione conjugates of fatty acid derivatives by plant glutathione transferases. J Biol Chem 284:21249-21256

Dixon DP, Skipsey M, Edwards R (2010) Roles for glutathione transferases in plant secondary metabolism. Phytochemistry $71: 338-350$

Duhoux A, Carrère S, Gouzy J, Bonin L, Délye C (2015) RNA-Seq analysis of rye-grass transcriptomic response to an herbicide inhibiting acetolactate-synthase identifies transcripts linked to non-target-site-based resistance. Plant Mol Biol 87:473-487

Eastmond PJ (2004) Glycerol-insensitive Arabidopsis mutants: gli1 seedlings lack glycerol kinase, accumulate glycerol and are more resistant to abiotic stress. Plant J 37:617-625

Farré G, Blancquaert D, Capell T, Van Der Straeten D, Christou P, Zhu C (2014) Engineering complex metabolic pathways in plants. Annu Rev Plant Biol 65:187-223

Fujita M, Hossain MZ (2003) Modulation of pumpkin glutathione S-transferases by aldehydes and related compounds. Plant Cell Physiol 44:481-490

Gaines TA, Lorentz L, Figge A, Herrmann J, Maiwald F, Ott M-C, Han H, Busi R, Yu Q, Powles SB, Beffa R (2014) RNA-Seq transcriptome analysis to identify genes involved in metabolismbased diclofop resistance in Lolium rigidum. Plant J 78:865-876

He H, Yu J, Chen G, Li W, He J, Li H (2012) Acute toxicity of butachlor and atrazine to freshwater green alga Scenedesmus obliquus and cladoceran Daphnia carinata. Ecotoxicol Environ Saf 80:91-96

Karavangeli M, Labrou NE, Clonis YD, Tsaftaris A (2005) Development of transgenic tobacco plants overexpressing maize glutathione S-transferase I for chloroacetanilide herbicides phytoremediation. Biomol Eng 22:121-128

Kawahigashi H (2009) Transgenic plants for phytoremediation of herbicides. Curr Opin Biotechnol 20:225-230

Kawahigashi H, Hirose S, Inui H, Ohkawa H, Ohkawa Y (2005) Enhanced herbicide cross-tolerance in transgenic rice plants coexpressing human CYP1A1, CYP2B6, and CYP2C19. Plant Sci 168:773-781

Kissoudis C, Kalloniati C, Flemetakis E, Madesis P, Labrou N, Tsaftaris A, Nianiou-Obeidat I (2015) Stress-inducible GmGSTU4 shapes transgenic tobacco plants metabolome towards increased salinity tolerance. Acta Physiol Plant 37:1-11

Kopka J, Schauer N, Krueger S, Birkemeyer C, Usadel B, Bergmüller E, Dörmann P, Weckwerth W, Gibon Y, Stitt M, Willmitzer L, Fernie AR, Steinhauser D (2005) GMD@CSB.DB: the Golm metabolome database. Bioinformatics 21:1635-1638

Krasensky J, Jonak C (2012) Drought, salt, and temperature stressinduced metabolic rearrangements and regulatory networks. J Exp Bot 63:1593-1608

Lechelt-Kunze C, Meissner RC, Drewes M, Tietjen K (2003) Flufenacet herbicide treatment phenocopies the fiddlehead mutant in Arabidopsis thaliana. Pest Manag Sci 59:847-856

Lydon J, Duke SO (1989) Pesticide effects on secondary metabolism of higher plants. Pestic Sci 25:361-373

Matsuda F, Yonekura-Sakakibara K, Niida R, Kuromori T, Shinozaki K, Saito K (2009) MS/MS spectral tag-based annotation of nontargeted profile of plant secondary metabolites. Plant Journal 57:555-577

Milligan AS, Daly A, Parry MAJ, Lazzeri PA, Jepson I (2001) The expression of a maize glutathione S-transferase gene in transgenic wheat confers herbicide tolerance, both in planta and in vitro. Mol Breed 7:301-315

Mochida K, Shinozaki K (2011) Advances in omics and bioinformatics tools for systems analyses of plant functions. Plant Cell Physiol 52:2017-2038

Nakabayashi R, Yonekura-Sakakibara K, Urano K, Suzuki M, Yamada Y, Nishizawa T, Matsuda F, Kojima M, Sakakibara H, Shinozaki K, Michael AJ, Tohge T, Yamazaki M, Saito K (2014) Enhancement of oxidative and drought tolerance in Arabidopsis by overaccumulation of antioxidant flavonoids. Plant J 77:367-379

Nobusawa T, Okushima Y, Nagata N, Kojima M, Sakakibara H, Umeda M (2013) Synthesis of very-long-chain fatty acids in the 
epidermis controls plant organ growth by restricting cell proliferation. PLoS Biol 11:e1001531

Orcaray L, Zulet A, Zabalza A, Royuela M (2012) Impairment of carbon metabolism induced by the herbicide glyphosate. J Plant Physiol 169:27-33

Park SW, Li W, Viehhauser A, He B, Kim S, Nilsson AK, Andersson MX, Kittle JD, Ambavaram MMR, Luan S, Esker AR, Tholl D, Cimini D, Ellerström M, Coaker G, Mitchell TK, Pereira A, Dietz KJ, Lawrence CB (2013) Cyclophilin 20-3 relays a 12-oxo-phytodienoic acid signal during stress responsive regulation of cellular redox homeostasis. Proc Natl Acad Sci USA 110:9559-9564

Powles SB, Yu Q (2010) Evolution in action: plants resistant to herbicides. Annu Rev Plant Biol 61:317-347

Schauer N, Steinhauser D, Strelkov S, Schomburg D, Allison G, Moritz T, Lundgren K, Roessner-Tunali U, Forbes MG, Willmitzer L, Fernie AR, Kopka J (2005) GC-MS libraries for the rapid identification of metabolites in complex biological samples. FEBS Lett 579:1332-1337

Serra AA, Nuttens A, Larvor V, Renault D, Couée I, Sulmon C, Gouesbet G (2013) Low environmentally relevant levels of bioactive xenobiotics and associated degradation products cause cryptic perturbations of metabolism and molecular stress responses in Arabidopsis thaliana. J Exp Bot 64:2753-2766

Shachar-Hill Y (2013) Metabolic network flux analysis for engineering plant systems. Curr Opin Biotechnol 24:247-255

Sharma S, Villamor JG, Verslues PE (2011) Essential role of tissuespecific proline synthesis and catabolism in growth and redox balance at low water potential. Plant Physiol 157:292-304

Skipsey M, Knight KM, Brazier-Hicks M, Dixon DP, Steel PG, Edwards R (2011) Xenobiotic responsiveness of Arabidopsis thaliana to a chemical series derived from a herbicide safener. J Biol Chem 286:32268-32276

Trenkamp S, Eckes P, Busch M, Fernie AR (2009) Temporally resolved GC-MS-based metabolic profiling of herbicide treated plants treated reveals that changes in polar primary metabolites alone can distinguish herbicides of differing mode of action. Metabolomics 5:277-291

Tzin V, Galili G (2010) New insights into the shikimate and aromatic amino acids biosynthesis pathways in plants. Mol Plant 3:956-972

Vila-Aiub MM, Neve P, Powles SB (2005) Resistance cost of a cytochrome P450 herbicide metabolism mechanism but not an ACCase target site mutation in a multiple resistant Lolium rigidum population. New Phytol 167:787-796

Voss I, Sunil B, Scheibe R, Raghavendra AS (2013) Emerging concept for the role of photorespiration as an important part of abiotic stress response. Plant Biol 15:713-722

Yu Q, Powles S (2014) Metabolism-based herbicide resistance and cross-resistance in crop weeds: a threat to herbicide sustainability and global crop production. Plant Physiol 166:1106-1118

Yuan JS, Tranel PJ, Stewart CN Jr (2007) Non-target-site herbicide resistance: a family business. Trends Plant Sci 12:6-13

Zabalza A, Orcaray L, Igal M, Schauer N, Fernie AR, Geigenberger P, van Dongen JT, Royuela M (2011) Unraveling the role of fermentation in the mode of action of acetolactate synthase inhibitors by metabolic profiling. J Plant Physiol 168:1568-1575

Zhang Y, Liu J (2011) Transgenic alfalfa plants co-expressing glutathione S-transferase (GST) and human CYP2E1 show enhanced resistance to mixed contaminates of heavy metals and organic pollutants. J Hazard Mater 189:357-362

Zhang Q, Xu F, Lambert KN, Riechers DE (2007) Safeners coordinately induce the expression of multiple proteins and MRP transcripts involved in herbicide metabolism and detoxification in Triticum tauschii seedling tissues. Proteomics 7:1261-1278

Zhang Y, Butelli E, De Stefano R, Schoonbeek HJ, Magusin A, Pagliarani C, Wellner N, Hill L, Orzaez D, Granell A, Jones JDG, Martin C (2013) Anthocyanins double the shelf life of tomatoes by delaying overripening and reducing susceptibility to gray mold. Curr Biol 23:1094-1100 